\title{
Visualizing HCV Core Protein via Fluorescent Unnatural Amino Acid Incorporation ${ }^{+}$
}

\author{
Leah Jane Fitzgerald Curnew ${ }^{1}$, Kate McNicholas ${ }^{1}$, Bridgette Green ${ }^{1}$, Jackie Barry ${ }^{1}$, Hannah L \\ Wallace 1, Lingyan Wang ${ }^{1}$, Cassandra Davidson ${ }^{1}$, John P Pezacki ${ }^{2}$ and Rodney S Russell ${ }^{1, *}$ \\ 1 Memorial University, Immunology and Infectious Diseases, Division of Biomedical Sciences, Faculty of \\ Medicine, St. John's, NL A1B 3V6, Canada; ljfc27@mun.ca (L.J.F.C.); kfm031@mun.ca (K.M.); \\ bpg453@mun.ca (B.G.); jpb243@mun.ca (J.B.); hlwallace@mun.ca (H.L.W.); lingyanw@mun.ca (L.W.); \\ cldavidson@mun.ca (C.D.) \\ 2 Department of Biochemistry, Microbiology and Immunology and Department of Chemistry and \\ Biomolecular Sciences, University of Ottawa, Ottawa, ON K1N 6N5, Canada; john.pezacki@uottawa.ca \\ * Correspondence: Rodney.Russell@med.mun.ca; Tel.: +1-709-864-2875 \\ † Presented at Viruses 2020-Novel Concepts in Virology, Barcelona, Spain, 5-7 February 2020.
}

Published: 14 July 2020

\begin{abstract}
Introduction: Unnatural amino acids (UAAs) share the same basic structure as proteinogenic amino acids. However, UAAs permit additional functions and applications to proteins due to their different side chains. Recent UAA applications include using fluorescent UAAs to label proteins. The UAA system provides an alternative method to traditional protein labeling mechanisms (antibodies, GFP, and tags, such as HA and HIS), which can affect protein functionality and topology. The purpose of this study was to visualize the hepatitis $\mathrm{C}$ virus (HCV) core protein using the fluorescent UAA Anap (3-[(6-acetyl-2-naphthalenyl)amino]-L-alanine). Methods: Huh-7.5 cells were co-transfected with $\mathrm{HCV}$ core plasmids containing amber stop codons at various positions throughout the coding sequence and a second plasmid encoding the orthogonal tRNA/synthetase pair that facilitates Anap incorporation. Three days post transfection, cells were stained for core protein and lipid droplets (LDs) and visualized using immunofluorescence or confocal microscopy. Results: We have optimized transfection protocols for the efficient expression of the tRNA/synthetase pair required for Anap incorporation and are able to visualize our core mutant proteins containing Anap. We have successfully substituted Anap into 11 different positions within the core, including substitutions for tryptophan, tyrosine, and phenylalanine residues. In addition, we have shown that our core mutants associate with cellular LDs, suggesting that the incorporation of the UAA did not disrupt core protein expression, stability, or cellular localization. Conclusions: We have demonstrated the establishment of a UAA incorporation system in an HCV protein without any obvious impact on core protein function. The ability to label viral proteins using fluorescent UAAs eliminates the requirement of antibodies or tags for protein visualization. In conclusion, the UAA system is a useful method to study HCV proteins and can potentially be used to label viruses for live cell and animal studies.
\end{abstract}

Keywords: unnatural amino acids; $\mathrm{HCV}$; $\mathrm{HCV}$ core protein; Anap; immunofluorescence microscopy; confocal microscopy

(C) 2020 by the authors. Licensee MDPI, Basel, Switzerland. This article is an open access article distributed under the terms and conditions of the Creative Commons Attribution (CC BY) license (http://creativecommons.org/licenses/by/4.0/). 